\title{
PRELIMINARY CALCULATION OF IRREGULAR TRIGUY MAST SUPPORT
}

\author{
KAZIMIERZ RYKALUK
}

Wrocław University of Environmental and Life Sciences, The Faculty of Environmental Engineering and Geodesy, ul. Grunwaldzka 55, 50-357 Wrocław, e-mail: kazimierz.rykaluk@up.wroc.pl

\begin{abstract}
The instantaneous tangential rigidity (corresponding to the applied horizontal load) of a triguy support with unequal ropes is determined. The differences between the ropes can be due to the different location, both horizontal and vertical, of their anchoring foundations and to the different types of ropes used. The horizontal rigidity of such a support has no general axis of symmetry. Therefore a formula for this rigidity in any direction and a formula for extreme rigidity angles are given in this paper. A notion of the guy's eigenvalue, standing for the initial tension force above which the guy participates effectively in the support's rigidity, is introduced. A numerical example is provided.
\end{abstract}

Key words: mast, irregular triguy support, initial tension values, forces in guys, numerical example

\section{INTRODUCTION}

When designing masts, a situation can be encountered when one or several of the following circumstances occur (the letter symbols are illustrated in Fig. 1):

- unequal length $s_{i}$ of guys at the same angle of chord inclination to level $\vartheta_{i}$ (due to the ground's inclination),

- unequal angle of inclination of chords relative to level $\vartheta_{i}$ (some of the guys have to be placed over the existing structures),

- unequal angles between chords in horizontal projection $\varphi_{i}$ (there are structures to be bypassed),

- unequal axial rigidities of ropes $E_{i} A_{i}$ (caused by repair conditions).

In such cases, an irregular triguy support, i.e., one which lacks initial horizontal axisymmetric rigidity and for which no directions of extreme horizontal rigidities can be determined in advance since they depend both on the initial tension values and the reaction values, is formed.

One should pay close attention to triguy supports since as the horizontal load on the mast increases in the conditions of insufficient initial tightening, their horizontal rigidity, particularly the lateral one (i.e., perpendicular to the wind load plane), decreases sharply as compared to the initial rigidity whereby the shaft may lose its stability.

It is always more difficult to calculate a mast with irregular multiguy supports than one with regu- lar supports, since apart from the iteration of the shaft's static solution (not to mention the complexity of the formulas) there is the additional iteration of the support's solution aimed at finding the directions of extreme rigidities.

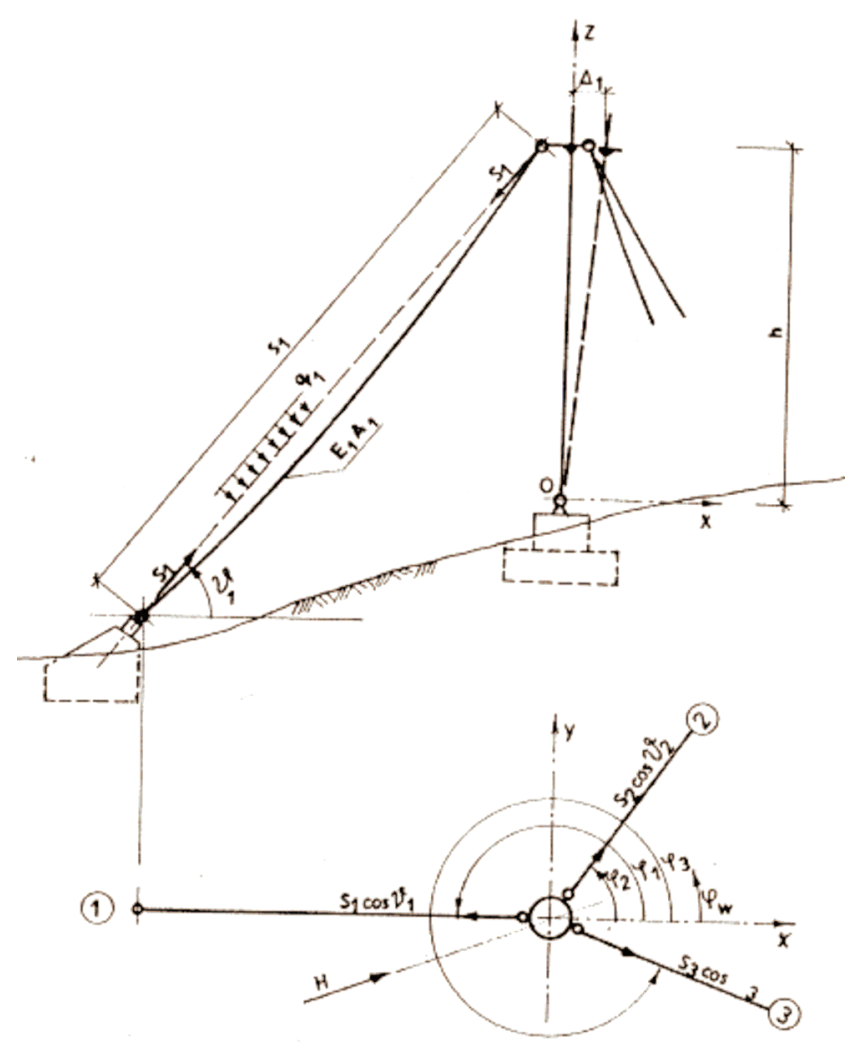

Fig. 1. A structural diagram of an irregular triguy support 
The symbols and the formulas used in this paper for a single guy were adopted after [1], while the forces in the ropes wee calculated by Petersen's method [2].

\section{INITIAL TENSION VALUES}

Initial tension values $S_{o i}(i=1,2,3)$, generally different from one another, must fulfil static conditions of equilibrium in the horizontal plane, i.e.,

$$
\sum_{i=1}^{3} S_{o i} \cos \vartheta_{\mathrm{i}} \cos \varphi_{\mathrm{i}}=0 ; \quad \sum_{i=1}^{3} S_{o i} \cos \vartheta_{\mathrm{i}} \sin \varphi_{\mathrm{i}}=0 .
$$

Up till now initial tension values for the ropes have been assumed according to construction recommendations [3], [6] and [7] which have a very general character. When they are assumed incorrectly, this prolongs the iterative design process. It is generally known that such initial tension values should be assumed for the adopted rope diameter that firstly, the greatest possible initial rigidity resulting in the shortening of the fundamental natural period, and secondly, sufficient operating rigidity in each direction are obtained. The guy's eigenvalue, standing for initial tension force $S_{o i}^{*}$ from which the horizontal displacement of the guy's upper hitching point starts diminishing for a fixed value of the support's horizontal reaction, is adopted as the initial tension reference level. Starting with the conditions for the positive determination of coefficient $K_{i}$ in the formula for the guy's horizontal displacement in the vertical plane, the sought eigenvalue for the upper hitching point will be obtained as the root of the following equation

$$
B_{i} S_{o i}^{* 3}-K_{T i} S_{o i}^{* 3}-A_{o i}=0
$$

where

$$
\begin{gathered}
A_{o i}=g_{o i}^{2} s_{i}^{3} \cos \vartheta_{\mathrm{i}} / 24, \\
B_{i}=s_{i} /\left(E_{i} A_{i} \cos \vartheta_{\mathrm{i}}\right), \\
K_{T i}=\varepsilon_{T l}\left(T_{l}-T_{o}\right) s_{i} / \cos \vartheta_{\mathrm{i}}-\varepsilon_{T t} h\left(T_{t}-T_{o}\right) \operatorname{tg} v_{\mathrm{i}},
\end{gathered}
$$

$g_{o i}$ - the rope's specific dead weight, $\mathrm{kN} / \mathrm{m}$,

$T_{l}, T_{t}, T_{o}$ - the operating temperature of the rope and the shaft, respectively, and the assembly temperature;

$\varepsilon_{T l}, \varepsilon_{T t}$ - coefficients of thermal expansions of the rope and the shaft, respectively.
For the range of climatic temperatures, equation (2) has only one real positive root. Its value can be determined most easily at assembly temperature since then

$$
S_{o i}^{* 3}=\left(A_{o i} / B_{i}\right)^{\frac{1}{3}} .
$$

In design practice, one can use eigenvalue $S^{*}{ }_{o i}$, calculated from formula (5) adding correction $\delta S^{*}{ }_{o i}$ for the rope's elongation due to the rise in temperature. On the basis of numerical calculations for guys made of type T. $1 * 61$ ropes [4], the following relation is proposed

$$
S_{o i}=\left(A_{o i} / B_{i}\right)^{\frac{1}{3}}+10^{4} \varepsilon_{T l}\left(T_{l}-T_{o}\right)\left(25 / d_{i}\right)^{0.42}\left(\operatorname{tg} \vartheta_{i}\right)^{0.8},
$$

where $d_{i}$ is the rope's catalog diameter in $\mathrm{mm}$.

After three eigenvalues for three ropes have been calculated, the following is determined

$$
S_{o, \min }>\max \left(S_{o i}^{*}\right) .
$$

The remaining two initial tension values must fulfil equations (1).

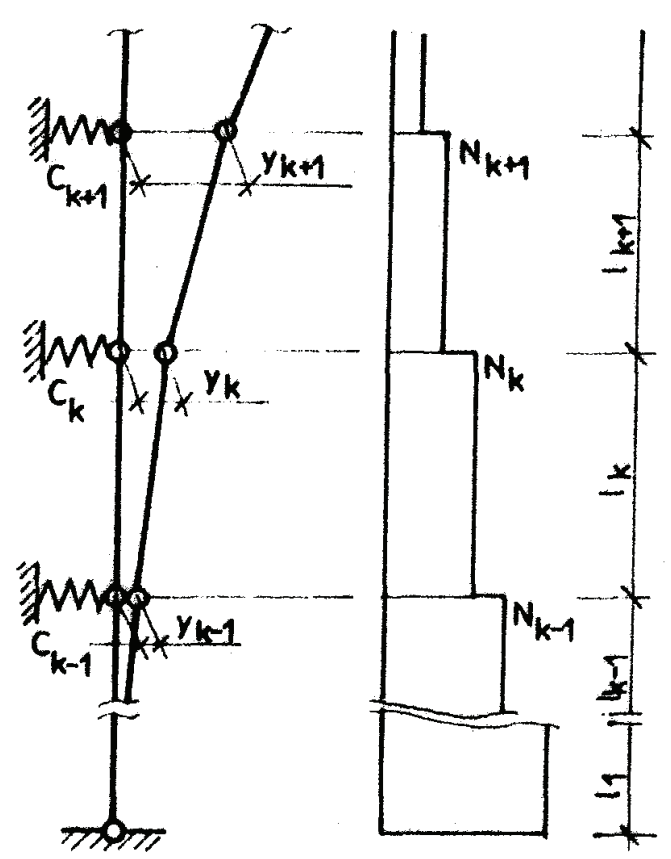

Fig. 2. The mast's shaft as a kinematic chain and a diagram of axial forces $N_{i}$

The support's minimum rigidity $C_{\min }$ in one of the extreme directions $\alpha_{\text {extr }}$, which is to be calculated, is the second criterion for the adoption of proper initial tension values. This rigidity must ensure the stability, in a bifurcation sense, of the shaft modeled by a kinematic chain (the low estimate). Let us use 
a linearized equation (the trigonometric functions of small arguments are replaced by the arguments themselves) of the $k$-th joint's equilibrium (Fig. 2)

$$
\begin{aligned}
N_{k} y_{k-1} / l_{k} & -\left(N_{k} / l_{k}+N_{k+1} / l_{k+1}-C_{p, k}\right) y_{k} \\
+ & N_{k+1} y_{k+1} / l_{k+1}=0 .
\end{aligned}
$$

Equations (8) written out for all the supports, after one parameter $\lambda$ has been introduced for all axial forces $N_{k}$, form a system of equations homogenous in respect of joints displacement $y_{k}$. From this system's solvability condition (the zero value of the main determinant) one obtains an algebraic equation in respect of critical load parameter $\lambda$ for the shaft with $n$ elastic supports

$$
\sum_{j=0}^{n} b_{j} \lambda^{n-j}=0
$$

where for a common case of a mast with four levels of guys (after omitting index min of $C$ ) we get

$$
\begin{aligned}
& b_{o}=1, \\
& b_{1}=-\left(C_{1}+C_{2}+C_{3}+C_{4}\right) l_{1} / N_{1}-\left(C_{2}+C_{3}+C_{4}\right) l_{2} / \\
& N_{2}-\left(C_{3}+C_{4}\right) l_{3} / N_{3}-C_{4} l_{4} / N_{4}, \\
& b_{2}=C_{1}\left(C_{2}+C_{3}+C_{4}\right) l_{1} l_{2} / N_{1} N_{2}+C_{2}\left(C_{3}+C_{4}\right) l_{2} l_{3} / \\
& N_{2} N_{3}+C_{3} C_{4} l_{3} l_{4} / N_{3} N_{4}+\left(C_{1}+C_{2}\right)\left(C_{3}+C_{4}\right) l_{1} l_{3} / \\
& N_{1} N_{3}+\left(C_{1}+C_{2}+C_{3}\right) C_{4} l_{1} l_{4} / N_{1} N_{4} \\
& +\left(C_{2}+C_{3}\right) C_{4} l_{2} l_{4} / N_{2} N_{4} \\
& b_{4}=-\left[C_{1} C_{2}\left(C_{3}+C_{4}\right) l_{1} l_{2} l_{3} / N_{1} N_{2} N_{3}\right. \\
& +C_{1} C_{4}\left(C_{2}+C_{3}\right) l_{1} l_{2} l_{4} / N_{1} N_{2} N_{4} \\
& +C_{3} C_{4}\left(C_{1}+C_{2}\right) l_{1} l_{3} l_{4} / N_{1} N_{3} N_{4} \\
& \left.+C_{2} C_{3} C_{4} l_{2} l_{3} l_{4} / N_{2} N_{3} N_{4}\right] \\
& b_{4}=C_{1} C_{2} C_{3} C_{4} l_{1} l_{2} l_{3} l_{4} / N_{1} N_{2} N_{3} N_{4}
\end{aligned}
$$

$\left(C_{4}=0\right.$ should be assumed for a mast with three levels of guys, whereas for a mast with two levels of guys one should adopt $C_{3}=C_{4}=0$ ). be

The support's initial rigidity in direction $\alpha$ will

$$
C_{o \alpha}=\sum_{i=1}^{3} C_{o i} \cos ^{2}\left(\varphi_{i}-\alpha\right)
$$

where $\varphi_{i}$ is the $i$-th guy's direction angle, whereas

$$
C_{o \alpha}=S_{o i}^{3} \cos \vartheta_{i} /\left(2 A_{o i}+B_{i} S_{o i}^{3}\right) \text {. }
$$

\section{FORCES IN GUY}

Using the conditions of the guy's equilibrium in horizontal plane $(x y)$, i.e.,

$$
\begin{aligned}
& H \cos \varphi_{w}+\sum_{i=1}^{3} S_{i} \cos \vartheta_{i} \cos \varphi_{i}=0, \\
& H \sin \varphi_{w}+\sum_{i=1}^{3} S_{i} \cos \vartheta_{i} \sin \varphi_{i}=0,
\end{aligned}
$$

where $H$ is the horizontal load on the joint, directed at angle $\varphi_{w}$ to axis $x$ which for reasons of convenience should be situated along guy No. 1, and the conditions of displacements inseparability (Fig. 3)

$$
\sum_{i=1}^{3} \Delta_{i}=0
$$

where

$$
\Delta_{i}=-A_{i} / S_{i}^{2}+B_{i} S_{i}-K_{i},
$$

the following algebraic septetic equation with unknown $S_{2}$ is obtained

$$
\sum_{k=0}^{7} a_{k} S_{2}^{(7-k)}=0
$$

in which

$$
\begin{aligned}
& a_{7}=-A_{2} S_{x y}^{2} S_{y}^{2}, \\
& a_{6}=-2 A_{2} S_{x y} S_{y} F_{1}, \\
& a_{5}=-\left(A_{1} S_{y}^{2}+A_{2} F_{2}+A_{3} S_{x y}^{2}\right) \\
& +\left(B_{1} S_{x y}-B_{3} S_{y}-K\right) S_{x y}^{2} S_{y}^{2}, \\
& a_{4}=-2\left(A_{1} S_{y} L+A_{2} F_{1} L M+A_{3} S_{x y} M\right) \\
& +\left[B_{1}\left(2 S_{x y}^{2} L+3 S_{x y} S_{y} M\right)+B_{2} S_{x y} S_{y}\right. \\
& \left.-B_{3}\left(2 S_{y}^{2} M+3 S_{x y} S_{y} L\right)-2 K F_{1}\right] S_{x y} S_{y}, \\
& a_{3}=-\left(A_{1} L^{2}+A_{2} L^{2} M^{2}+A_{3} M^{2}\right)+B_{1} S_{x y} F_{3} \\
& -2 B_{2} S_{x y} S_{y} F_{1}-B_{3} S_{y} F_{4}-K F_{2}, \\
& a_{2}=B_{1} M F_{4}+B_{2} F_{2}-B_{3} L F_{3}-2 K L M F_{1}, \\
& a_{1}=\left[B_{1}\left(3 S_{x y} L M+2 S_{y} M^{2}\right)+2 B_{2} F_{1}\right. \\
& \left.-B_{3}\left(3 S_{y} L M+2 S_{x y} L^{2}\right)\right] L M-K L^{2} M^{2}, \\
& a_{0}=\left[B_{1} M+B_{2}-B_{3} L\right) L^{2} M^{2},
\end{aligned}
$$




$$
\begin{aligned}
& F_{1}=S_{x y} L+S_{y} M, \\
& F_{2}=S_{x y}^{2} L^{2}+S_{y}^{2} M^{2}+4 S_{x y} S_{y} L M, \\
& F_{3}=S_{x y}^{2} L^{2}+3 S_{y}^{2} M^{2}+6 S_{x y} S_{y} L M, \\
& F_{4}=3 S_{x y}^{2} L^{2}+S_{y}^{2} M^{2}+6 S_{x y} S_{y} L M, \\
& K=K_{1}+K_{2}+K_{3}, \quad[\mathrm{~m}], \\
& A_{i}=q_{i}^{2} s_{i}^{3} /\left(24 \cos \vartheta_{i}\right), \quad\left[\mathrm{kN}^{2} / \mathrm{m}\right] \\
& K_{i}=-A_{o i} / S_{o i}^{2}+B_{i} S_{o i}-K_{T i}, \quad[\mathrm{~m}]
\end{aligned}
$$

$q_{i}=\left[\left(g_{o i} \cos \vartheta_{i}+q_{v i}\right)^{2}+q_{H i}^{2}\right]^{\frac{1}{2}}-$ the load perpendicular to the $i$-th guy's chord, $\mathrm{kN} / \mathrm{m}$;

$$
\begin{aligned}
& q_{v i}=W_{i} \sin \vartheta_{i} \cos \left(\varphi_{i}+\varphi_{w}\right), \\
& q_{H i}=W_{i} \sin \left(\varphi_{i}+\varphi_{w}\right), \\
& W_{i}=-q_{w i}\left[1-\cos ^{2} \vartheta_{i} \cos ^{2}\left(\varphi_{i}+\varphi_{w}\right)\right]^{1 / 2}, \\
& q_{w i}=\gamma_{f} q_{k} C_{e} C_{x} d_{i} \beta-\text { the wind load on a } d_{i} \text { diameter }
\end{aligned}
$$
guy at the height of $2 h_{i} / 3$, according to [6], $\mathrm{kN} / \mathrm{m}$;

$$
\begin{gathered}
L=\cos \vartheta_{2} \sin \varphi_{2} /\left(\cos \vartheta_{3} \sin \varphi_{3}\right), \\
M=\left(\cos \vartheta_{2} \cos \varphi_{2}-L \cos \vartheta_{3} \cos \varphi_{3}\right) / \cos \vartheta_{1}, \\
S_{x y}=\left(H \cos \varphi_{w}-S_{y} \cos \vartheta_{3} \cos \varphi_{3}\right) / \cos \varphi_{1}, \\
S_{y}=H \sin \varphi_{w} /\left(\cos \vartheta_{3} \sin \varphi_{3}\right),
\end{gathered}
$$

$q_{k}-$ the characteristic wind velocity pressure.

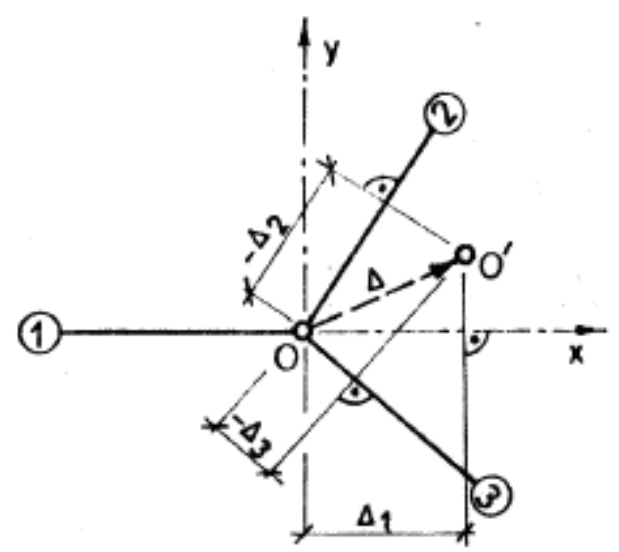

Fig. 3. The horizontal displacement of the support by vector $\Delta_{1}$

Equation (15) has at least one real root which follows from a fundamental theorem of algebra [5]. Numerous numerical calculations done for real systems show that there are three real roots of which only one is positive. Its value is in the interval

$$
\left[1+\left(|c| / a_{7}\right)^{1 / k_{1}}, 1+\left(\left|a_{7}\right| / a_{0}\right)^{1 / k_{2}}\right],
$$

where $|c|$ - the absolute value of the highest coefficient of negative polynomial $\left(\sum a_{k} S_{2}^{7-k}\right)$ and $\left(-\sum a_{k} S_{2}^{k}\right)$, respectively.

Taking into account force $S_{2}$ and expressions (16)-(19), the forces in the remaining ropes are calculated from the following conditions of equilibrium

$$
S_{1}=S_{x y}+S_{2} M ; \quad S_{3}=-S_{y}-S_{2} L .
$$

The $i$-th guy's instantaneous rigidity $C_{i}$ is calculated from formula (11) in which simply index " $O$ " is removed and the value calculated from formula (16) substituted for $A_{o i}$. The overall rigidity of the support in direction $\alpha$ is calculated in an analogous way, using formula (10).

The criterion condition for the extremum of function $C_{i}$ yields

$$
\begin{gathered}
\alpha_{\text {extr }}=0.5\left[\operatorname{arctg}\left(\sum_{i=1}^{3} C_{i} \sin 2 \varphi_{i} / \sum_{i=1}^{3} C_{i} \cos 2 \varphi_{i}\right)+k \pi\right], \\
(k=0,1) .
\end{gathered}
$$

\section{NUMERICAL EXAMPLE}

An irregular triguy support at the temperature of assembly and under wind action in direction $\varphi_{w}=0^{\circ}$ is to be solved for the following geometry

$$
\begin{gathered}
h=92 \mathrm{~m}, \\
\varphi_{1}=180^{\circ}, \quad \varphi_{2}=72^{\circ}, \quad \varphi_{3}=320^{\circ}, \\
\vartheta_{1}=45^{\circ}, \quad \vartheta_{2}=60^{\circ}, \quad \vartheta_{3}=50^{\circ}, \\
s_{1}=130 \mathrm{~m}, \quad s_{1}=120 \mathrm{~m}, \quad s_{1}=140 \mathrm{~m} .
\end{gathered}
$$

All the guys are made of type $\mathrm{T} 1 * 61$ rope of $d=25 \mathrm{~mm}$ diameter, for which $A=375 \mathrm{~mm}^{2}, g_{o}=$ $0.0315 \mathrm{kN} / \mathrm{m}$ and $E=145 \mathrm{GPa}$.

Using (18) and (19), we calculate:

$$
L=-1.150, \quad M=1.019 \text {. }
$$

The guys' characteristics calculated from formulas (3) and their eigenvalues calculated from formula (5) are as follows

$$
\begin{gathered}
A_{01}=62.658 ; \quad A_{02}=36.864 ; \quad A_{03}=71.148 ; \\
B_{1}=3.381 \cdot 10^{-3} ; \quad B_{2}=4.414 \cdot 10^{-3} ; \quad B_{3}=4.004 \cdot 10^{-3} ; \\
S_{01}^{*}=26.463 \mathrm{kN} ; \quad S_{02}^{*}=20.289 \mathrm{kN} ; S_{03}^{*}=26.095 \mathrm{kN} .
\end{gathered}
$$


Table 1. Initial tension values $S_{\alpha i}$ of the guys and their initial rigidities $C_{o i}$

\begin{tabular}{|c|c|c|c|c|c|c|c|c|c|}
\hline No. & $S_{01}$ & $S_{02}$ & $S_{03}$ & $C_{01}$ & $C_{02}$ & $C_{03}$ & $K_{1}$ & $K_{2}$ & $K_{3}$ \\
\hline \multicolumn{5}{|c|}{$\mathrm{kN}$} & \multicolumn{3}{|c|}{$\mathrm{kN} / \mathrm{m}$} \\
\hline 1 & 13.2 & 12.95 & 14.90 & 12.22 & 13.04 & 13.66 & -0.315 & -0.162 & -0.261 \\
\hline 2 & 26.46 & 25.97 & 29.86 & 69.71 & 57.95 & 68.80 & 0 & 0.060 & 0.040 \\
\hline 3 & 50 & 49.05 & 56.40 & 161.31 & 99.23 & 134.04 & 0.144 & 0.201 & 0.203 \\
\hline 4 & 100 & 98.10 & 112.80 & 201.67 & 111.31 & 157.71 & 0.332 & 0.429 & 0.446 \\
\hline
\end{tabular}

From formula (7) we obtain: $S_{0, \min }=S_{01}=26.463 \mathrm{kN}$. The calculations were done for all four values of initial tightening $S_{01}=13.2,26.463,50$ and $100 \mathrm{kN}$, (the first value was assumed for comparison with the obtained rigidity of the support, measured by the horizontal displacement at given reaction). Forces $S_{02}$ and $S_{03}$ were calculated from formulas (1), initial rigidities $C_{o i}$ of the ropes along their horizontal directions from formulas (11), and coefficients $K_{i}$ from formula (17) into which $K_{T i}=0$ should be put since a design situation at the temperature of assembly is considered. The calculated values have been compiled in table 1.

The directions and the extreme initial rigidities calculated using formulas (23) and (10), respectively, have been gathered in Table 2 .

Table 2. Extreme rigidities $C_{\max }$ and $C_{\min }$ of the support considered and the corresponding directions

\begin{tabular}{|c|c|c|c|c|}
\hline$S_{01}$ & $\alpha_{\text {extr }}^{\max }$ & $\alpha_{\text {extr }}^{\min }$ & $C_{o \alpha}^{\max }$ & $C_{o \alpha}^{\min }$ \\
\hline $\mathrm{kN}$ & \multicolumn{2}{|c|}{ grades } & \multicolumn{2}{|c|}{$\mathrm{kN} / \mathrm{m}$} \\
\hline 13.2 & $-27^{\circ} 32^{\prime} 45^{\prime \prime}$ & $62^{\circ} 27^{\prime} 15^{\prime \prime}$ & 22.994 & 15.934 \\
\hline 26.4 & $-22^{\circ} 02^{\prime} 53^{\prime \prime}$ & $67^{\circ} 57^{\prime} 07^{\prime \prime}$ & 122.451 & 76.039 \\
\hline 50 & $-17^{\circ} 37^{\prime} 01^{\prime \prime}$ & $72^{\circ} 22^{\prime} 59^{\prime \prime}$ & 261.140 & 133.439 \\
\hline 100 & $-16^{\circ} 19^{\prime} 01^{\prime \prime \prime}$ & $73^{\circ} 43^{\prime} 59^{\prime \prime}$ & 317.269 & 152.413 \\
\hline
\end{tabular}

It was assumed that the support considered would carry horizontal force $H=20,40$, and $80 \mathrm{kN}$, depending on the span length of the shaft's spans adjacent to the support, the type of the shaft's structure and the installations mounted on the shaft.

Figure 4 illustrates the distribution of the support's horizontal rigidity $C_{\alpha}$ for all the initial tension values $S_{01}$ and loads $H$, while Fig. 5 shows the relationship between force $S 1$ and displacement $\Delta_{1}$, depending on load $H$ for fixed initial tension values $S_{01}$ (Fig. 5a) and on initial tension $S_{01}$ for fixed forces $H$ (Fig. 5b).

To make the diagrams in Fig. 4a more legible, the distributions of initial rigidity $C_{o \alpha}$ have been plotted in the figure's upper part, whereas those of current rigidity $C_{\alpha}$ under initial tightening $S_{01}=13.2$ and $100 \mathrm{kN}$ (for the initial tightening of $100 \mathrm{kN}$ all curves for
$H=20,40$ and $80 \mathrm{kN}$ practically coincide) have been grouped in its lower part. Figure $4 \mathrm{~b}$ again shows diagrams $C_{o \alpha}$ in its top part and the distributions of current rigidity $C_{\alpha}$ under initial tightenings $S_{01}=26.4$ and $50 \mathrm{kN}$ in its bottom part.

Changes in the maximum and minimum rigidities in relation to the initial rigidities depending on joint load $H$ are illustrated in Fig. 6.

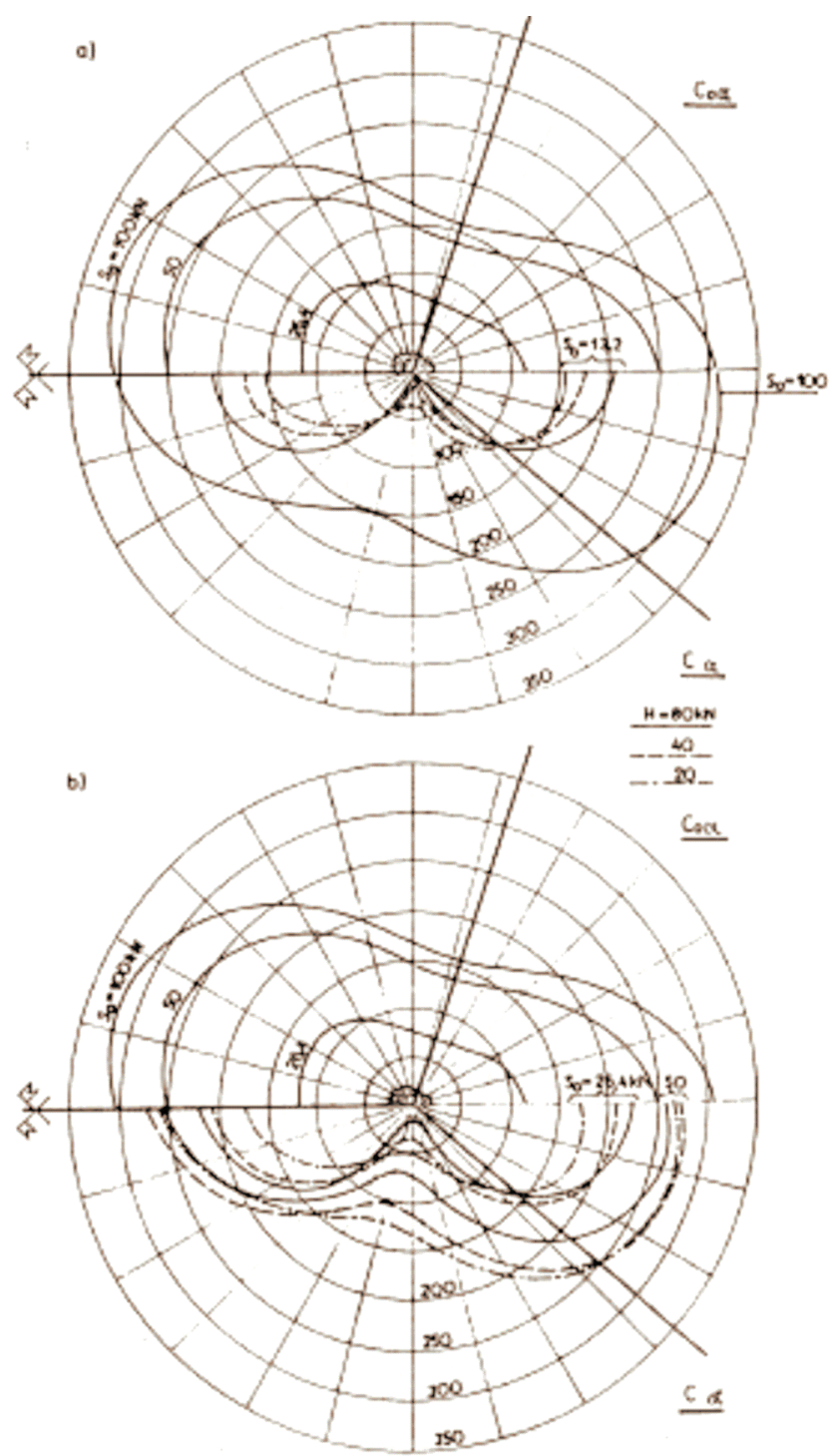

Fig. 4. The distribution of horizontal initial $C_{o \alpha}$ and current $C_{\alpha}$ rigidity (description in the text) 
a)

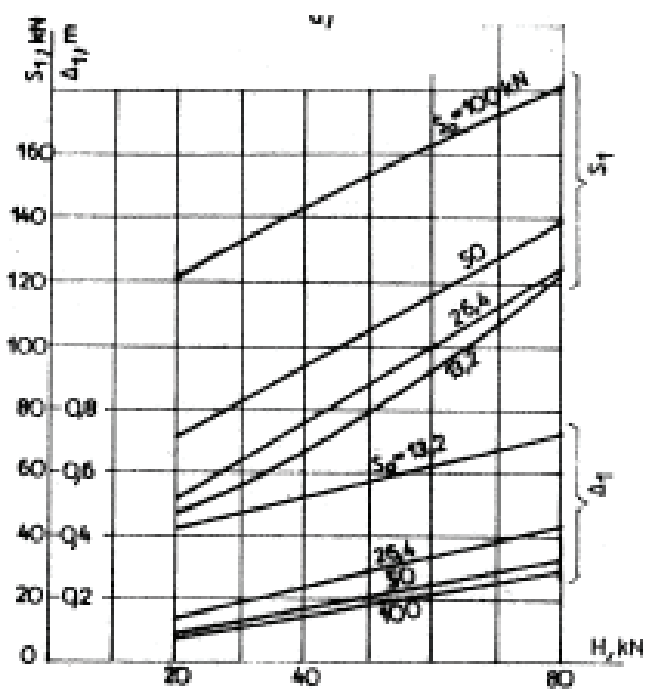

b)

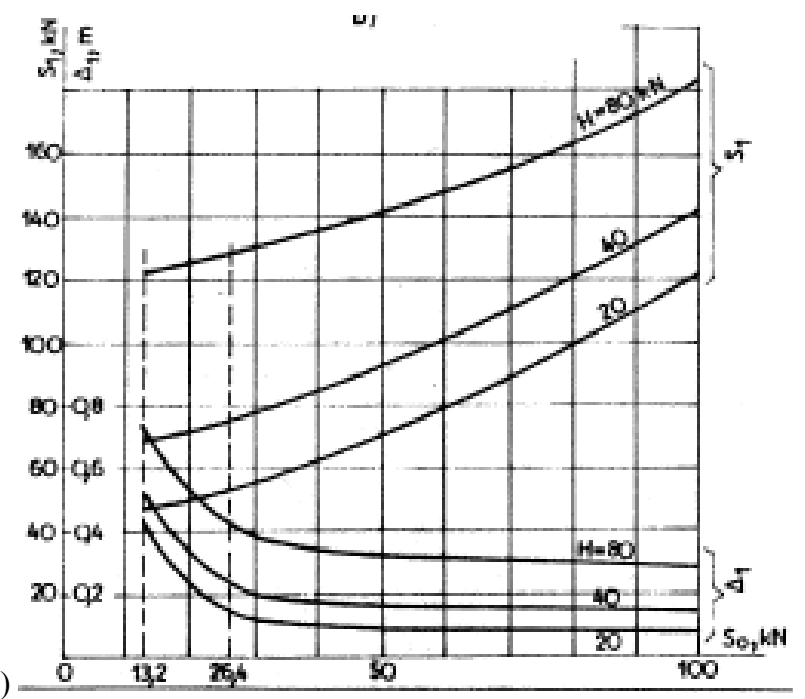

Fig. 5. The relationship between force $S_{1}$ in the first rope, the shaft's horizontal displacement $\Delta_{1}$ in this rope's plane and: (a) horizontal load $H$ on the joint under different initial tension values $S_{0}$,

(b) initial tension $S_{0}$ of the rope under different horizontal loads $H$ on the joint

a)

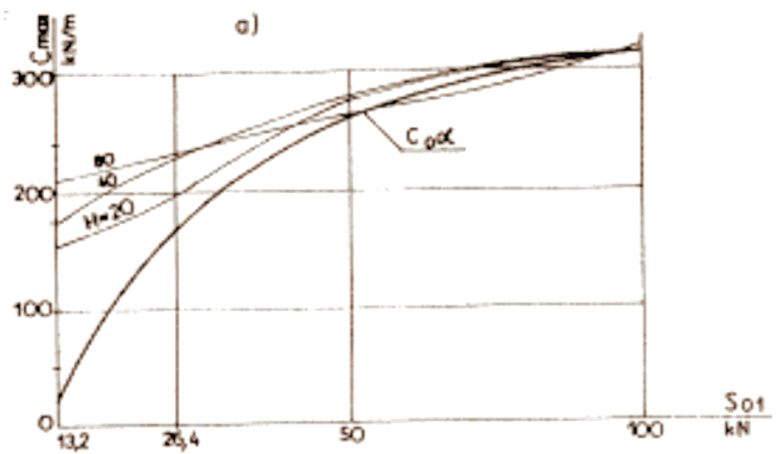

b)

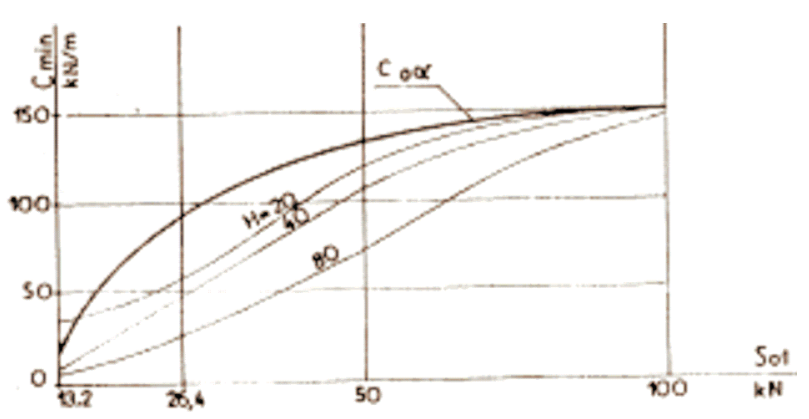

Fig. 6. Changes in the extreme rigidities for the support considered

\section{CONCLUSIONS}

The formulas derived in this paper allow one to calculate the characteristics of any triguy support. The force of initial tightenings is the basic parameter of the support's rigidity. The initial tension of the least tightened rope in the support should not be lower than the guy's eigenvalue calculated from formula (5). Otherwise, such a support is very sensitive to horizontal load $H$. This manifests itself mainly in a wide variation of the support's rigidity in the plane of loading, which increases the number of iterations in the shaft's static solution, and in a sharp decrease in the rigidity in the lateral plane whereby the shaft may lose its stability.

\section{REFERENCES}

[1] Grochowski I., Radio and Television Masts, Guide the designer of metal structures, Arkady, Warsaw 1982, Vol. 2, 471-508, (in Polish).

[2] Petersen Ch., Stahlbau, 3 Auflage, Vieweg, Wiesbaden 1993.

[3] Kozıowski T., Steel Radio and Television Masts and Towers, Arkady, Warsaw 1965, (in Polish).

[4] EN 12385-10: 2008 Steel wire ropes-safety. Part 10: Spiral ropes for general structural applications.

[5] Guide engineer and construction technician, Mathematics, Arkady, Warsaw 1977, Vol. 1/1, (in Polish).

[6] EN 1993-3-1: 2006 Design of steel structures. Part 3-1: Towers, masts and chimneys - Towers and masts.

[7] PaŁKowski Sz., Structures with tension components, Wydawnictwa Naukowo-Techniczne, Warsaw 1994, (in Polish). 